\title{
The use of real-time digital video in the assessment of post- operative outcomes of breast reconstruction
}

\author{
Gilmour $A^{a}$, Mackay IR ${ }^{a}$, Young $D^{b}$, Hill ME ${ }^{a}$, Brown La, Malyon A $^{a}$ \\ ${ }^{a}$ Canniesburn Plastic Surgery Unit, Glasgow Royal Infirmary, 84 Castle Street, Glasgow, G4 0SF \\ ${ }^{\mathrm{b}}$ Department of Mathematics \& Statistics, University of Strathclyde, Glasgow
}

\section{Introduction}

The ideal goal of breast reconstruction is to replace the removed breast tissue with something similar and to achieve symmetry with the contralateral breast. ${ }^{1}$

Conventional methods of assessing the aesthetic outcome of any form of breast surgery have often involved the use of questionnaires and still clinical photographs shown to an assessment panel. These two-dimensional images provide a limited amount of information to the assessor. To obtain further information more complex measuring tools; such as the use of three dimensional imaging, have recently been described. ${ }^{2,3}$

All these techniques provide static results and lack the ability to confer any information about the effect which movement has on the reconstructed breast. Gui et al previously highlighted this point and felt that better assessment may be made using video clips, but noted that there were no studies comparing the use of still photographs with video footage. ${ }^{4}$

\section{Aim of the Study}

The aim of this study was to assess whether standardised real-time digital video footage was a valid assessment tool in assessing post-operative outcomes and to compare its use against standardised static photography.

\section{Materials \& Methods}

Ethical approval for this study was granted by the West of Scotland REC1.

No standard protocol exists for the use of real time digital video capture in the assessment of postoperative breast reconstruction outcomes. As such a script was created by the senior author (AM) outlining the instructions for a sequence of movements (incorporating all views obtained by photography), deemed necessary to allow subjective assessment. (SEE IMAGE) 


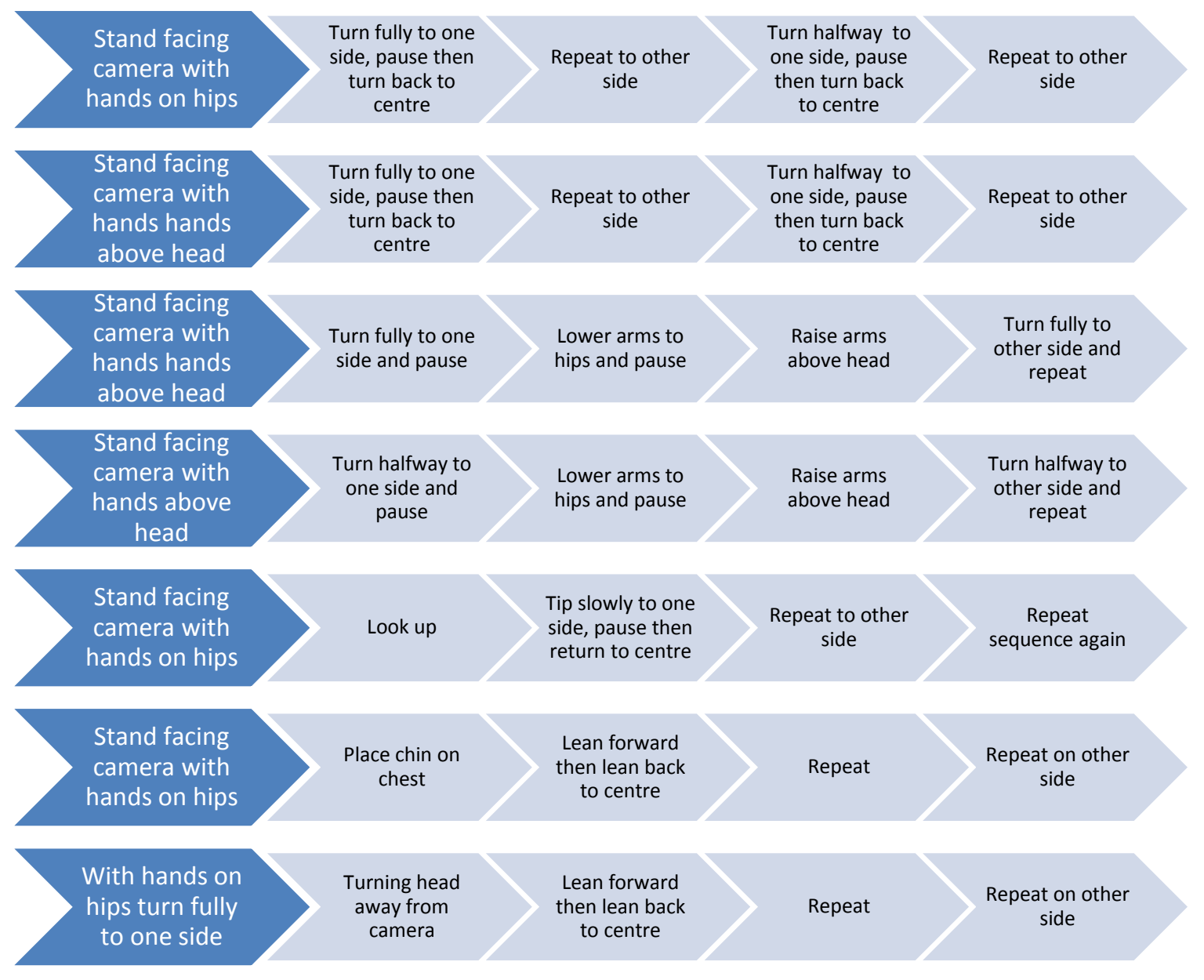

The services of a female model were utilised to perform the above scripted routine whilst undergoing digital video capture. Narrated position and movement instructions were then added to the video footage to create an instructional demonstrational digital video clip providing both clear visual and audio instructions to the viewer. (SEE VIDEO)

Thirty-five patients at least six months post unilateral breast reconstruction were recruited and consented for this study from our treatment database. Prior to consent the potential study subjects were shown the instructional demonstrational digital video clip to ensure that they were fully informed as to what would be expected.

Patients were initially asked to complete a 22 item questionnaire developed by Stanton et al with regards to their opinion on their reconstruction. ${ }^{5}$ This questionnaire requires the patient to compare their reconstruction with the non-reconstructed breast and to score on a simple 1 (no difference) - 4 (large difference) scale for various aspects ranging from cosmesis to function.

Study subjects were then shown the instructional video clip again and asked to perform the same movements in time with the narrated video whilst undergoing digital video capture. Each video clip ranged approximately 140-180 seconds in duration.

As per current practice within our unit these patients also had 6 standardised digital photographs taken of their reconstruction (frontal view with hands at sides, frontal view with hands raised above 
head, right lateral, left lateral, left oblique and right oblique) (SEE IMAGE). One patient set of photographs was lost during the capture process and this patient was not included in the photography assessment process below.

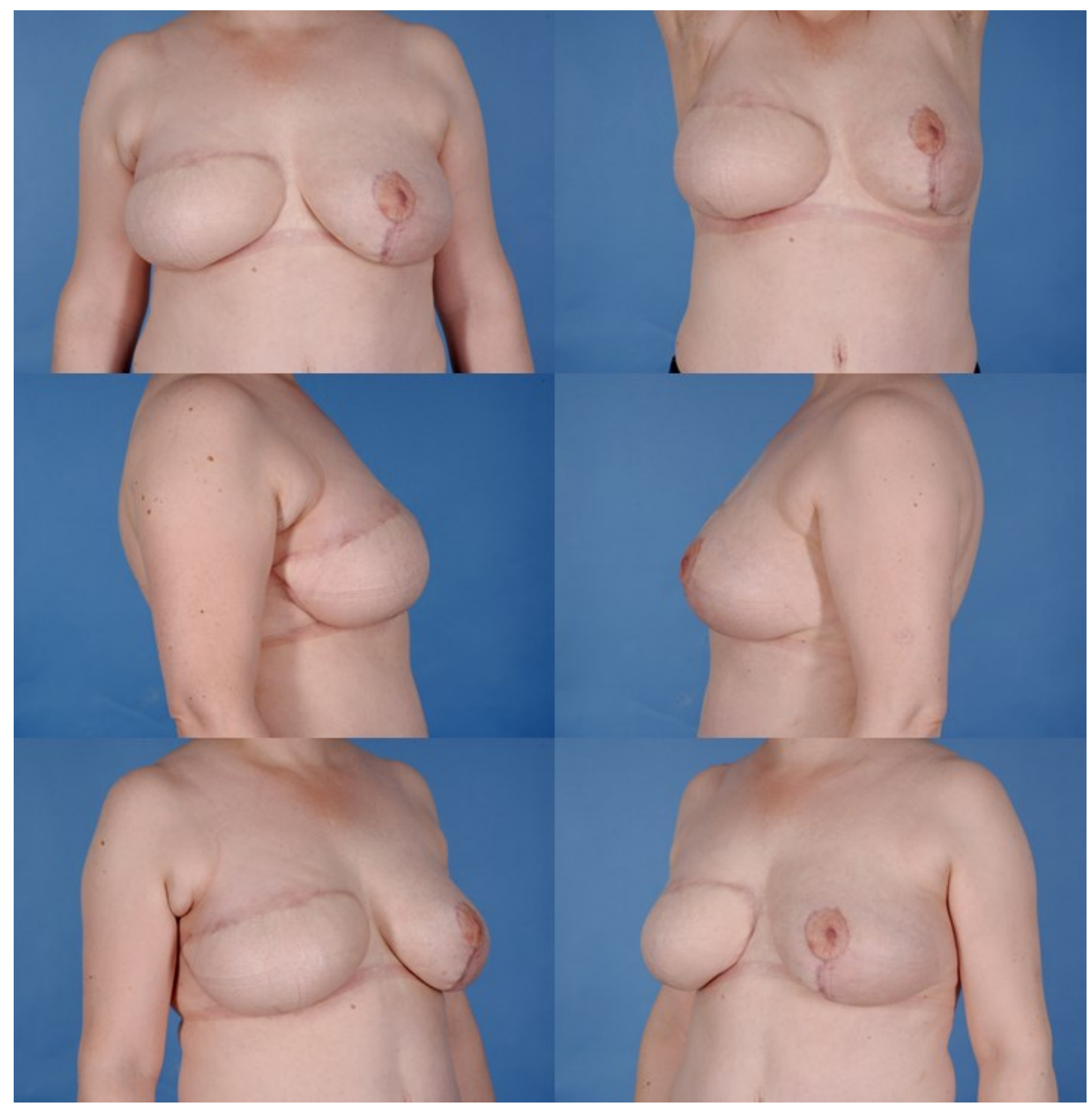

Each six individual patient digital photographs were compiled and placed onto a single Microsoft PowerPoint slide and a complete PowerPoint presentation consisting of all patient photographs was created. The same process was utilised for each patient video in a separate presentation. The order of the image slides and video clip slides were then randomised.

A panel of 21 assessors was assembled consisting of 7 Plastic Surgeons (6 Consultant Plastic Surgeons all with specialist interest in breast and 1 senior onco-plastic breast fellow); 5 General Breast Surgeons and 9 Non-surgical allied health professional staff.

The panel members were shown each presentation in turn and asked to rate their overall opinion of each reconstruction (in comparison to the contralateral breast) based upon the still photographs or 
video presentation on a simple 1-4 scale (SEE TABLE) as originally devised by Harris. ${ }^{6}$ In addition the panel was also asked to rank their opinion on individual aspects of each reconstruction in terms of Size, Shape and Elevation using a similar 1-4 scale as developed by Stanton et al. (See Table)

Panel members were blinded as to the identity of each patient at all stages. At the end of the assessment process each panel member was also asked to provide their opinion on whether they found the still photographs or video clips more useful/informative in assessing the post-operative outcome of breast reconstruction.

\begin{tabular}{|c|l|}
\hline \multicolumn{2}{|c|}{ Breast Assessment Score } \\
\hline $\mathbf{1}$ & Excellent - Treated breast nearly identical to contralateral breast \\
\hline $\mathbf{2}$ & Good - Treated breast slightly different than contralateral breast \\
\hline $\mathbf{3}$ & Fair - Treated breast clearly different from contralateral breast \\
\hline $\mathbf{4}$ & Poor - Treated breast seriously distorted in comparison to contra-lateral breast \\
\hline
\end{tabular}

Panel inter-rater agreement was calculated using Kendall's Coefficient of Concordance for both still photographs and video assessments. The values obtained can then be interpreted from a range of 0 (no agreement) to 1 (complete agreement).

Overall median panel photograph and video scores for each patient were compared to the patient questionnaire self-scores relating to cosmesis (size, shape and elevation) using a Spearmans rank correlation test to assess levels of agreement between panel and patient.

\section{$\underline{\text { Results }}$}

Of the 35 patients who took part in this study, 15 underwent a unilateral breast reconstruction using an extended Autologous Latissimus Dorsi (ALD) flap, 12 had free tissue transfer reconstructions (11 DIEP flaps, 1 TRAM flap); 5 had ALD flap + Implant and 3 had implant only based reconstructions. 21 reconstructions were left sided compared to 14 right.

The mean age of the 35 patients who took patient in this study was 52 years old (range $35-67$ ).

The average file storage size of each photo and video set were 0.38 megabytes and 14.9 megabytes respectively.

\begin{tabular}{|c|c|c|c|c|}
\hline \multicolumn{5}{|c|}{ Kendall's Coefficient of Concordance Scores for Total Panel } \\
\cline { 2 - 5 } & Photographs & $p$ & Video & $p$ \\
\hline Overall & 0.507 & 0.00 & 0.548 & 0.00 \\
\hline Size & 0.514 & 0.00 & 0.514 & 0.00 \\
\hline Shape & 0.486 & 0.00 & 0.505 & 0.00 \\
\hline Elevation & 0.514 & 0.00 & 0.514 & 0.00 \\
\hline
\end{tabular}


Overall there was a "moderate" degree of inter-rater agreement amongst the panel in all categories. However there was a slightly greater degree of panel inter-rater reliability using video assessment in comparison to photographs to assess the overall cosmesis of the reconstruction ( $0.55 \mathrm{vs} 0.51$ ) and to assess shape (0.49 vs 0.51 ). There was no difference in levels of panel inter-rater agreement between the two methods in terms of assessing size and elevation ( 0.51 vs 0.51 ).

\section{Kendall's Coefficient of concordance Scores for Panel Assessment}

\begin{tabular}{|r|c|c|c|c|c|c|c|c|}
\hline & \multicolumn{2}{|c|}{ Overall } & \multicolumn{2}{c|}{ Size } & \multicolumn{2}{c|}{ Shape } & \multicolumn{2}{c|}{ Elevation } \\
\cline { 2 - 9 } & Photo & Video & Photo & Video & Photo & Video & Photo & Video \\
\hline Plastic Surgery Panel & 0.65 & 0.64 & 0.64 & 0.68 & 0.55 & 0.60 & 0.48 & 0.56 \\
\hline General Surgery Panel & 0.55 & 0.56 & 0.56 & 0.51 & 0.52 & 0.48 & 0.42 & 0.44 \\
\hline Other Panel & 0.50 & 0.57 & 0.50 & 0.53 & 0.52 & 0.58 & 0.38 & 0.40 \\
\hline Total Panel & 0.51 & 0.55 & 0.51 & 0.51 & 0.49 & 0.51 & 0.51 & 0.51 \\
\hline
\end{tabular}

\begin{tabular}{|c|c|}
\hline \multicolumn{2}{|c|}{ Interpretation of Kendall's W scores } \\
\hline W Score & Level of agreement \\
\hline 0.1 & Very weak agreement \\
\hline 0.3 & Weak Agreement \\
\hline 0.5 & Moderate agreement \\
\hline 0.7 & Strong agreement \\
\hline 0.9 & Unusually strong agreement \\
\hline
\end{tabular}

On dividing the panel into their respective categories (plastic surgeon, general surgeon and nonsurgical) the highest degree of inter-rater agreement (moderate-strong) occurs amongst the expert plastic surgeon panel utilising both photographs and video images. Interestingly the plastic surgery panel showed a slightly lower level of agreement using video footage to assess the "overall" quality of the reconstruction ( 0.65 vs 0.64 ) but actually agreed more using video to assess the individual components of the reconstruction (size, shape and elevation).

\begin{tabular}{|c|c|c|c|c|}
\hline \multicolumn{5}{|c|}{$\begin{array}{c}\text { Spearmans' Rank Correlation of Panel Scores } \\
\text { Compared to Patient Scores }\end{array}$} \\
\hline & \multicolumn{4}{|c|}{ Overall } \\
\hline & Photographs & $\mathrm{p}$ & Videos & $\mathrm{p}$ \\
\hline Plastic Surgery Panel & 0.217 & 0.204 & 0.282 & 0.091 \\
\hline General Surgery Panel & 0.357 & 0.032 & 0.466 & 0.004 \\
\hline Other Panel & 0.083 & 0.629 & 0.346 & 0.036 \\
\hline Total Panel & 0.281 & 0.097 & 0.311 & 0.061 \\
\hline
\end{tabular}


On comparing the patient self-scores obtained in comparison to the median panel scores using both photography and video assessment it is evident that there is a greater degree of correlation between the patient and the panel using video footage as assessment material. The General Surgery panel showed the greatest degree of correlation with the patients' own views using both photography and video footage to assess. The other (non-surgical) panel showed the least degree of correlation using the still photographs as the assessment medium but showed a huge increase in correlation when using the video footage ( 0.083 vs 0.346 ).

All 21 members (100\%) of the panel were of the opinion that video clip footage was more useful in terms of assessing the post-operative outcome of breast reconstruction and that it yielded more information than the still photograph images.

\section{Discussion}

Reconstructions based solely upon restoration of "form"; such as breast, are more difficult to assess in terms of outcome as the aesthetic result is largely subjective. This is in contrast to assessment of reconstructions based solely upon restoration of "function"; such as tendon repair / reconstruction, which often have quantitative outcomes. Overall patient satisfaction is the most important factor in determining the success of any form of surgery dealing with aesthetics. ${ }^{7}$ This complicates matters further as it has been shown that patients overall subjective assessment as to the success of their reconstruction is due to far more than their cosmetic result ${ }^{8}$

Still photography coupled with panel assessment has been widely utilised in many studies for the purposes of assessment of cosmesis post breast conserving surgery or reconstruction. ${ }^{6,9-16}$ However the design; in terms of methodology, of such studies vary greatly as shown in a recent systematic review. ${ }^{17}$ There have also been concerns that these methods lack precision and are difficult to reproduce. ${ }^{9}$

In an effort to improve upon the flaws described above, other more objective assessment methods have been described such as the use of computer software designed to take objective measurements from two dimensional digital photographs with subsequent computer analysis of various indices linked to symmetry. ${ }^{18-20}$ This has been further improved upon with the use of threedimensional imaging systems; allowing the capture of a greater amount of information. ${ }^{2,3}$ The objective data obtained from three-dimensional imaging system shows promise in being more accurate than traditional subjective assessments. ${ }^{21}$ However all these methods require specialist software, expertise and /or equipment not routinely utilised in most plastic surgery units. They also all rely upon static imagery and offer no impression as to the effects of movement on a reconstructed breast.

When Gui et al originally theorised that video footage may provide a more accurate assessment than static photographs alone they foresaw several problems in terms of specialist equipment, cost, storage space and standardisation. ${ }^{4}$ Since then technology, software and storage media have advanced significantly as well as becoming cheaper. As a result these issues are no longer as constraining as previously highlighted. Whilst the average file size associated with the digital video clips in this study (14.9 megabytes) was 39.2 times greater than that of the digital photograph files 
(0.38megabytes) it is still relatively small in comparison to the capacity of most modern storage media which often have gigabytes or terabytes of space. The model demonstration video which was created for the purposes of this study proved very effective in terms of providing a standardised routine for the patients to emulate. One concern raised after the completion of this study was that each digital video clip was too long at between 140-180 seconds. This was in part due to the fact that the original demonstration script duplicated each of the movements twice. It is feasible that the length of the video clips can easily be reduced to half the duration by omitting the duplication of each of these movements and creating a more streamlined instructional video. This would confer several benefits in that; the video capture process would quicker; assessment time shorter and the file size of all video clips would be significantly reduced.

Many published articles analysing ordinal data such as those result obtained in this study have focussed on utilising Kappa scores to assess inter-rater reliability. ${ }^{9,15,19-21}$ These kappa scores however only measure the level of "absolute agreement" between raters and fail to take into account the magnitude of difference observed using ordinal data. The Kendall Coefficient of Concordance is a better measure of agreement for ordinal responses as it calculates the consistency amongst raters by also taking the ranking of the scores into account. ${ }^{22}$ Furthermore several of the papers have changed their assessment scales by dichotomising categories of the scale to obtain higher kappa scores which invariably skews the results. ${ }^{20,21}$

All 21 members of the assessment panel felt that video footage yielded a greater amount of information upon which to base their assessment. It was readily apparent throughout the assessment process that the video clips were capturing information which would not be apparent on standardised photographs. In particular these included significant "muscle twitching" of innervated Latissimus Dorsi flaps whilst moving the arm and also the relative absence of reconstruction movement in patients with implant only based reconstructions.

The total panel results show that; whilst marginal, video is equivalent or better than still photographs in terms of inter-rater reliability. The Plastic surgeon sub-division of the panel had the highest degree of inter-rater reliability and this may reflect their expertise in terms of reconstruction outcomes.

Given that patient satisfaction is the goal in any form of reconstruction it is of importance to have an assessment tool which more accurately correlates with patient self-evaluation. Our results show that digital video footage assessment gives significantly better correlation between patient views and panel views in comparison to still photograph assessment. This again could be due to the fact that it offers a greater degree of information with regards to the reconstruction (such as mobility) which is not apparent on static photographs. It is also apparent that the greatest degree of correlation between patient perception and sub-panel (in both photograph and video clip assessment) occurred with the General Breast Surgeons. This is interesting and may be worth further investigation.

\section{Conclusion}

From our initial findings we believe that real time digital video footage coupled with panel review is a valid method of assessing post-operative outcomes of breast reconstruction and appears superior to still photographs in terms of inter-rater agreement and correlation with patient self-assessment. 


\section{Conflict of interest}

The assessment panel evening was supported by BMI Healthcare and Mentor ${ }^{\circledR}$ Medical UK.

\section{Funding}

This study was funded by awards obtained from the Stephen Plumpton Charitable Trust Fund and the Canniesburn Research Trust. 


\section{References}

1. Godfrey PM, Godfrey NV, Romita MC. Restoring the Breast to Match the Normal Side. Annals of Plastic Surgery 1993;31:392-8.

2. Henseler $\mathrm{H}$, Khambay BS, Bowman A, et al. Investigation into accuracy and reproducibility of a 3D breast imaging system using multiple stereo cameras. J Plast Reconstr Aesthet Surg 2011;64:577-82.

3. Henseler $\mathrm{H}$, Smith J, Bowman A, et al. Objective evaluation of the latissimus dorsi flap for breast reconstruction using three-dimensional imaging. J Plast Reconstr Aesthet Surg 2012;65:120915.

4. Gui GP, Kadayaprath G, Tan SM, et al. Evaluation of outcome after immediate breast reconstruction: prospective comparison of four methods. Plast Reconstr Surg 2005;115:1916-26. 5. Stanton AL, Krishnan L, Collins CA. Form or function? Part 1. Subjective cosmetic and functional correlates of quality of life in women treated with breast-conserving surgical procedures and radiotherapy. Cancer 2001;91:2273-81.

6. Harris JR, Levene MB, Svensson G, Hellman S. Analysis of cosmetic results following primary radiation therapy for stages I and II carcinoma of the breast. Int J Radiat Oncol Biol Phys 1979;5:25761.

7. Ching S, Thoma A, McCabe RE, Antony MM. Measuring Outcomes in Aesthetic Surgery: A Comprehensive Review of the Literature. Plastic and Reconstructive Surgery 2003;111:469-80.

8. Beesley $\mathrm{H}, \mathrm{Ull}$ mer $\mathrm{H}$, Holcombe $\mathrm{C}$, Salmon P. How patients evaluate breast reconstruction after mastectomy, and why their evaluation often differs from that of their clinicians. J Plast Reconstr Aesthet Surg 2012;65:1064-71.

9. Cardoso MJ, Cardoso J, Santos AC, Barros H, Cardoso de Oliveira M. Interobserver agreement and consensus over the esthetic evaluation of conservative treatment for breast cancer. Breast 2006;15:52-7.

10. Gui GP, Tan SM, Faliakou EC, Choy C, A'Hern R, Ward A. Immediate breast reconstruction using biodimensional anatomical permanent expander implants: a prospective analysis of outcome and patient satisfaction. Plast Reconstr Surg 2003;111:125-38.

11. Mosahebi A, Ramakrishnan V, Gittos M, Collier J. Aesthetic outcome of different techniques of reconstruction following nipple-areola-preserving envelope mastectomy with immediate reconstruction. Plast Reconstr Surg 2007;119:796-803.

12. Asplund $\mathrm{O}$, Nilsson B. Interobserver variation and cosmetic result of submuscular breast reconstruction. Scand J Plast Reconstr Surg 1984;18:215-20.

13. Sacchini V, Luini A, Tana S, et al. Quantitative and qualitative cosmetic evaluation after conservative treatment for breast cancer. Eur J Cancer 1991;27:1395-400.

14. Edsander-Nord A, Brandberg Y, Wickman M. Quality of life, patients' satisfaction, and aesthetic outcome after pedicled or free TRAM flap breast surgery. Plast Reconstr Surg 2001;107:1142-53.

15. Charfare H, MacLatchie E, Cordier C, et al. A comparison of different methods of assessing cosmetic outcome following breast- conserving surgery and factors influencing cosmetic outcome. British Journal of Medical Practitioners 2010;3:310-5.

16. Gahm J, Jurell G, Edsander-Nord A, Wickman M. Patient satisfaction with aesthetic outcome after bilateral prophylactic mastectomy and immediate reconstruction with implants. J Plast Reconstr Aesthet Surg 2010;63:332-8.

17. Potter $\mathrm{S}$, Harcourt $\mathrm{D}$, Cawthorn $\mathrm{S}$, et al. Assessment of cosmesis after breast reconstruction surgery: a systematic review. Ann Surg Oncol 2011;18:813-23.

18. Fitzal F, Krois $\mathrm{W}$, Trischler $\mathrm{H}$, et al. The use of a breast symmetry index for objective evaluation of breast cosmesis. Breast 2007;16:429-35. 
19. Heil J, Dahlkamp J, Golatta M, et al. Aesthetics in breast conserving therapy: do objectively measured results match patients' evaluations? Ann Surg Oncol 2011;18:134-8.

20. Cardoso MJ, Cardoso J, Amaral N, et al. Turning subjective into objective: the BCCT.core software for evaluation of cosmetic results in breast cancer conservative treatment. Breast 2007;16:456-61.

21. Henseler H, Smith J, Bowman A, et al. Subjective versus objective assessment of breast reconstruction. J Plast Reconstr Aesthet Surg 2013;66:634-9.

22. Siegel S, Castellan NJ. Nonparametric Statistics for The Behavioral Sciences. 2nd ed. New York: McGraw-Hill; 1988. 